\title{
$\mathrm{BMC}$
}

Systems Biology

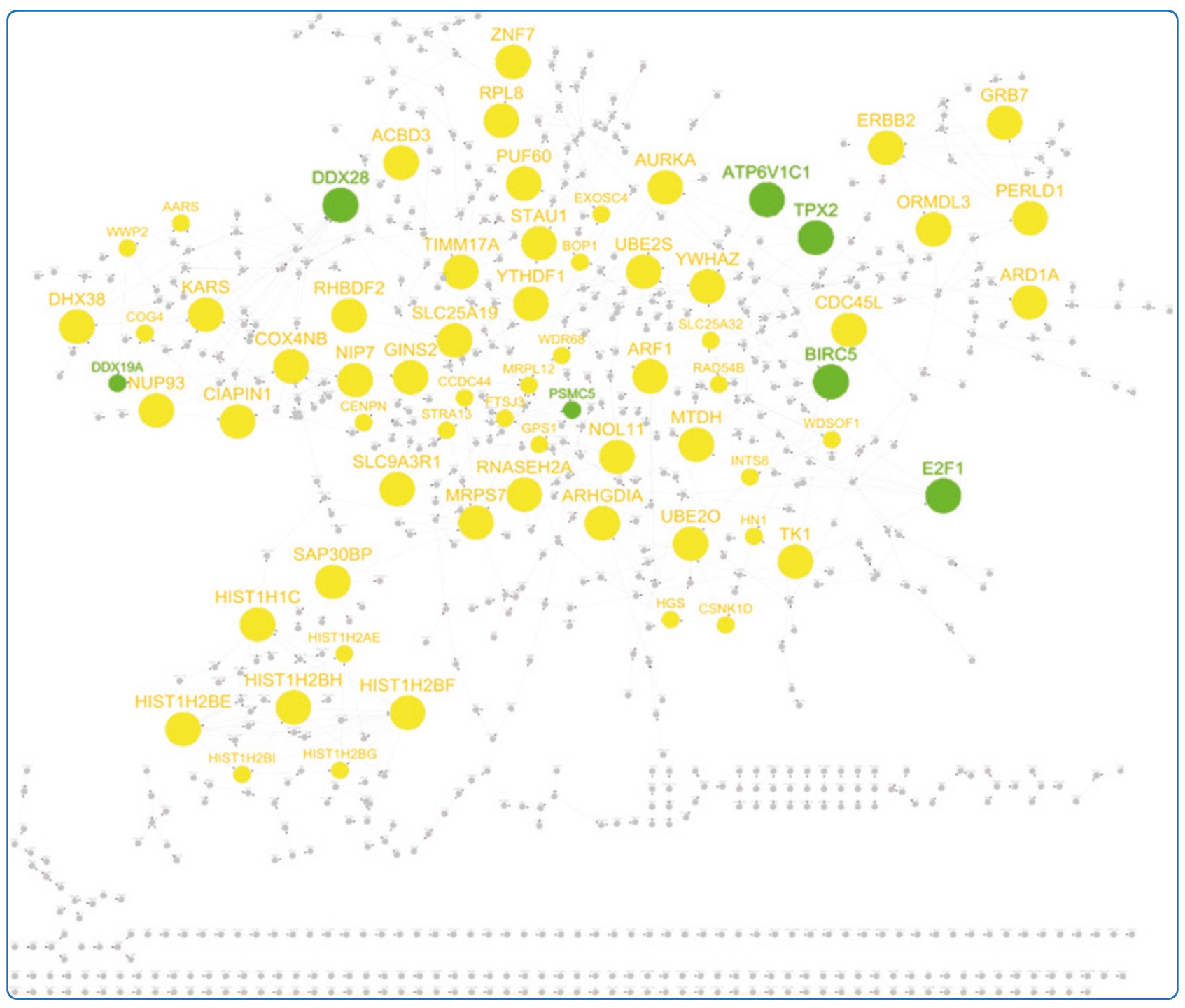

\section{Inferring causal genomic alterations in breast cancer using gene expression data}

Tran et al. 


\title{
Inferring causal genomic alterations in breast cancer using gene expression data
}

\author{
Linh M Tran ${ }^{1,2 \dagger}$, Bin Zhang ${ }^{1,2^{*} \dagger}$, Zhan Zhang ${ }^{2}$, Chunsheng Zhang ${ }^{2}$, Tao Xie ${ }^{2}$, John R Lamb ${ }^{2}$, Hongyue Dai ${ }^{2}$, \\ Eric E Schadt ${ }^{1,2,3^{*}}$ and Jun Zhu ${ }^{1,2^{*}}$
}

\begin{abstract}
Background: One of the primary objectives in cancer research is to identify causal genomic alterations, such as somatic copy number variation (CNV) and somatic mutations, during tumor development. Many valuable studies lack genomic data to detect CNV; therefore, methods that are able to infer CNVs from gene expression data would help maximize the value of these studies.

Results: We developed a framework for identifying recurrent regions of CNV and distinguishing the cancer driver genes from the passenger genes in the regions. By inferring CNV regions across many datasets we were able to identify 109 recurrent amplified/deleted CNV regions. Many of these regions are enriched for genes involved in many important processes associated with tumorigenesis and cancer progression. Genes in these recurrent CNV regions were then examined in the context of gene regulatory networks to prioritize putative cancer driver genes. The cancer driver genes uncovered by the framework include not only well-known oncogenes but also a number of novel cancer susceptibility genes validated via siRNA experiments.

Conclusions: To our knowledge, this is the first effort to systematically identify and validate drivers for expression based CNV regions in breast cancer. The framework where the wavelet analysis of copy number alteration based on expression coupled with the gene regulatory network analysis, provides a blueprint for leveraging genomic data to identify key regulatory components and gene targets. This integrative approach can be applied to many other large-scale gene expression studies and other novel types of cancer data such as next-generation sequencing based expression (RNA-Seq) as well as CNV data.
\end{abstract}

Keywords: breast cancer, copy number variation, gene regulatory networks, oncogenes

\section{Background}

Tumors arise from the activation of oncogenes along with the inactivation of tumor suppressor genes via somatic gene mutations or copy number variation $(\mathrm{CNV})$. Identification of the genetic/genomic changes that drive biological processes associated with cancer onset or progression assists in the development of therapeutics targeting the affected proteins or their downstream consequences[1-4]. Although extensive gene expression studies have been conducted for identifying tumor signature genes associated with poor outcome $[5,6]$, the reproducibility of these signatures is low $[7,8]$,

\footnotetext{
*Correspondence: zhangb@gmail.com; eric.schadt@gmail.com; jun. zhu@sagebase.org

+ Contributed equally

'Sage Bionetworks, Seattle, WA 98109, USA

Full list of author information is available at the end of the article
}

posing a major challenge for identifying the causal genetic/genomic variations.

Genome-wide DNA copy number variation (CNV) has been increasingly used for identifying biomarkers and targets in cancer research[9-11]. Unfortunately, the CGH, SNP genotype, and DNA sequencing data typically used to detect $\mathrm{CNV}$ are not available for many published, large-scale studies. Therefore, the development of methods to infer CNV from non-genetic data collected in these studies would serve to enhance their scientific value. Strong correlations between CNV and gene expression have been observed[11,12] and initially suggested the possibility of detecting $\mathrm{CNV}$ directly from gene expression data. Recently, the Analysis of Copy number alteration by Expression (ACE) algorithm was developed to identify amplified or deleted chromosome
C Biomed Central

(c) 2011 Tran et al; licensee BioMed Central Ltd. This is an Open Access article distributed under the terms of the Creative Commons Attribution License (http://creativecommons.org/licenses/by/2.0), which permits unrestricted use, distribution, and reproduction in any medium, provided the original work is properly cited. 
regions based on gene expression data[13]. While this approach demonstrated the utility of leveraging expression data to identify candidate $\mathrm{CNV}$ regions and genes whose expressions might be affected by the candidate $\mathrm{CNV}$, the identified regions were often large and harbored many genes. Furthermore, no objective mechanism was employed to distinguish cancer driver genes from passenger genes within a putative $\mathrm{CNV}$ region.

Here we describe a novel Wavelet based Analysis of Copy number alteration by Expression (WACE) algorithm and combine it with Bayesian networks via a key driver analysis to provide a systematic and unified approach for distinguishing cancer driver genes from passenger genes residing in inferred copy number variation sites.

\section{Methods}

\section{Preprocessing data}

We collected four independent breast cancer datasets, NKI[14], Wang[6], Miller[15], and Christos[8]. NKI samples were profiled on the Agilent Human $25 \mathrm{~K}$ platform comprised of 24,496 non-control oligonucletoide probes while the other studies were carried out using the Affymetrix HG133A platform, compromised of 22,282 probe sets. Each of the four microarray datasets was adjusted for estrogen and progesterone receptor (ER/PR) status as well as age to avoid their influence. The data were fit using a robust linear regression model (rlm function from $\mathrm{R}$ statistical package), and the residuals with respect to the model fit were carried forward in all subsequence analyses as the gene expression traits. In all analyses except ACE, the expression of individual gene/probe was used. In the subsequent analysis, for a gene with multiple probes we used the average expression profile to represent its expression so as to eliminate the weight effect due to multiple data points on a single location.

We also downloaded the gene expression and aCGH data from the Stanford University Breast Cancer Study http://smd.stanford.edu/[16]. Again the expression profiles of multiple probes representing the same gene were consolidated by average. The expression and aCGH data were adjusted for array batch, ER/PR status and age to avoid their influence.

\section{A Unified Framework for Expression based CNV Inference and Causal Gene Identification}

We developed a framework for integrating CNV inference and gene regulatory network analysis to provide a systematic and unified approach to prioritizing genes residing in inferred CNV (ICNV) regions (Figure 1). Under this framework, we first developed a waveletbased ACE algorithm (WACE) to more efficiently and accurately detect amplified/deleted regions of the

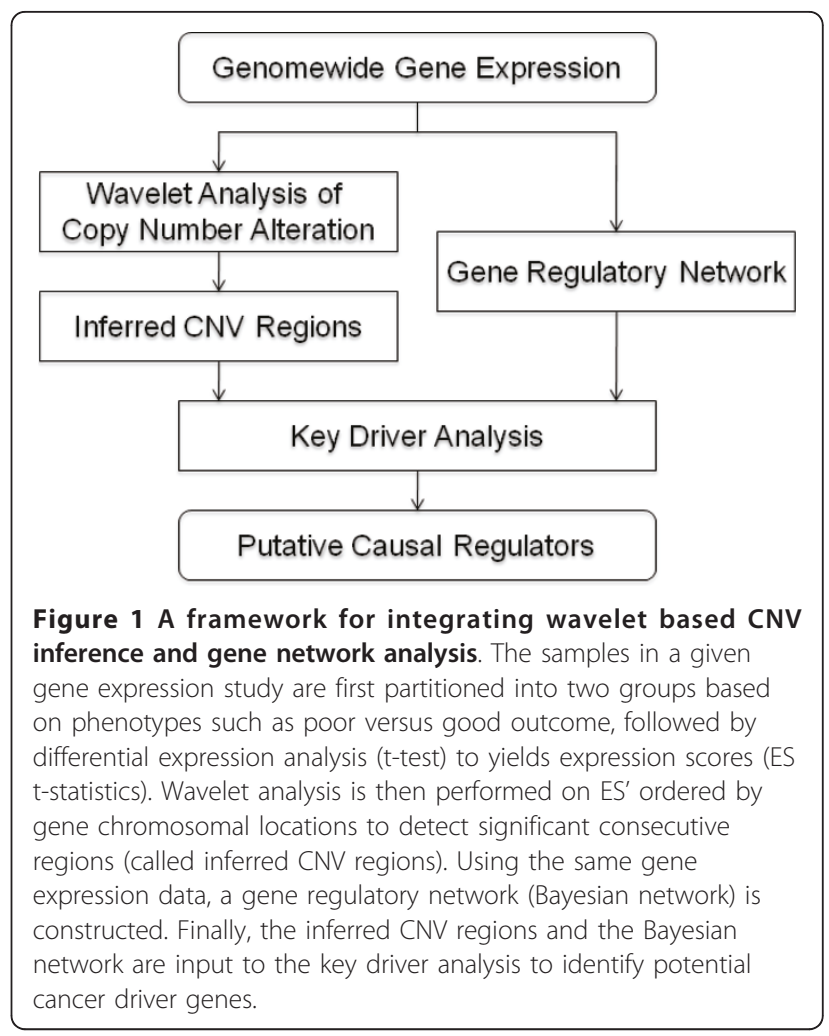

genome in cancer samples. For a given gene expression study the samples are first classified into two groups based on phenotypes such as poor versus good outcome and the two corresponding subsets of gene expression data are then taken as input for WACE to infer significantly amplified or deleted CNV regions. Meanwhile, a regulatory network is constructed using the Bayesian network reconstruction method. Finally, genes on the inferred CNV regions are input into a key driver analysis to identify hub genes in the network as potential cancer driver genes. When multiple datasets are available, all ICNV regions are aligned to form recurrent $\mathrm{CNV}$ regions and multiple regulatory networks are combined into a single network. The recurrent $\mathrm{CNV}$ regions and the combined network then go through the key driver analysis to identify putative regulators.

\section{Wavelet based Analysis of Copy number alteration by Expression (WACE)}

WACE algorithm is described in detail in the Method of Additional File 1 and summarized by a diagram in Figure 2. In brief, for a given dataset, the gene expression traits were first ordered according to their physical location on chromosomes. The expression scores (ES, t-statistics) were computed for each gene with respect to the good and bad tumor outcome, and were then subjected to wavelet transform to obtain the neighboring scores (NS). To evaluate the significance of the NS's on each 


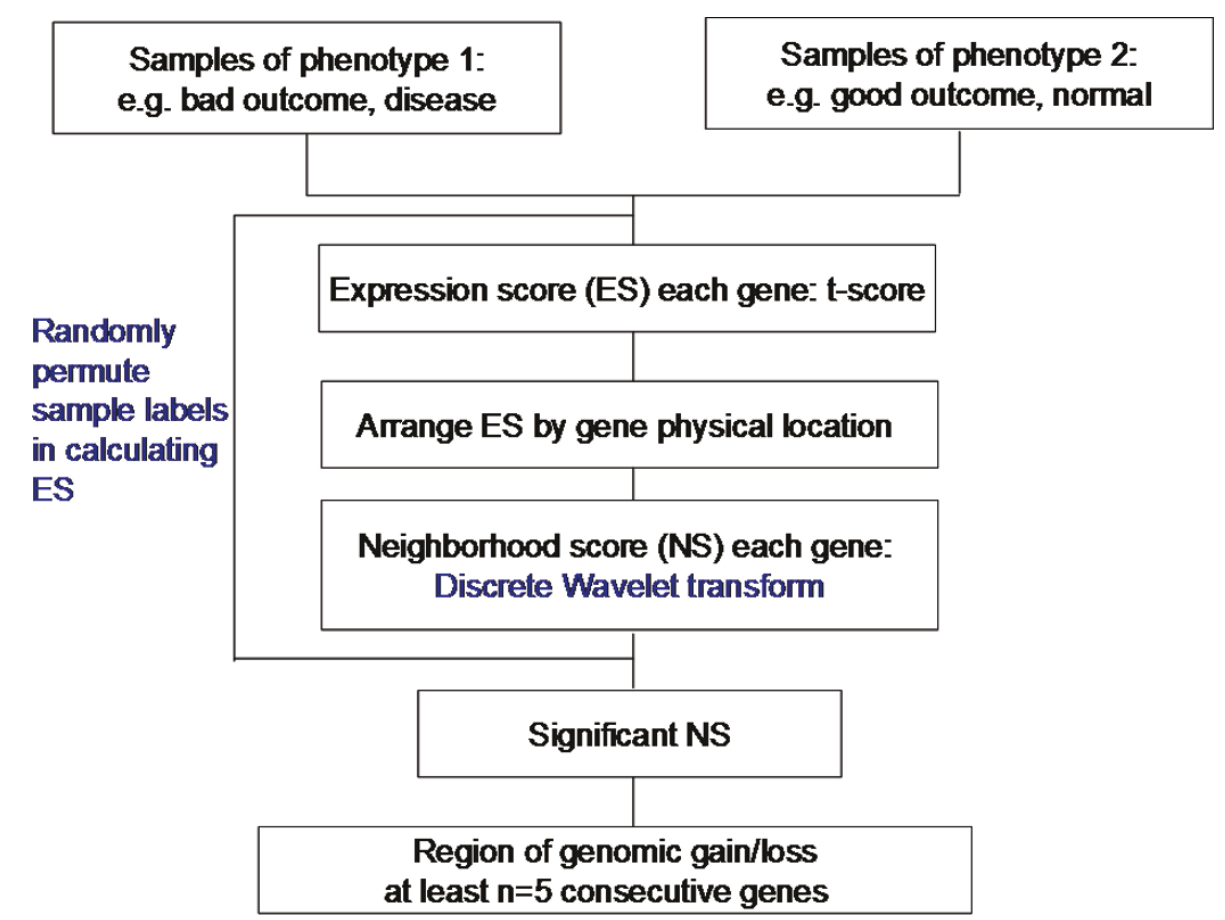

Figure 2 Outline of the WACE algorithm. For a given gene expression dataset, the samples are classified into two groups based on phenotypes such as poor versus good outcome and the genes are ordered based on their physical location on chromosomes. Expression scores (ES, t-statistics) for all the genes are computed and then subjected to wavelet transform to obtain smoothed ES, called neighboring score (NS). The significance (false discovery rate, FDR) of NS on each individual chromosome is empirically approximated based on its null distribution by performing the same wavelet transform on "random" ES's based on the randomized samples. A segment containing at least $n$ consecutive positive/negative NS with FDR $\leq 0.01$ is defined as an inferred CNV region. ICNV regions from multiple datasets are finally aligned to determine the recurrent regions of CNV.

individual chromosome, we empirically approximated its null distribution by applying wavelet transform to "random" ES's based on randomizing the sample class labels with respect to the expression vectors, repeating this process 1000 times. The false discovery rate for each observed NS was computed as the fraction of random NS's that were greater than (less than) or equal to the observed value if NS $>0(\mathrm{NS}<0)$. After evaluating the statistical significance of NS, an ICNV region on a chromosome was identified if it harbored at least $n$ consecutive positive/negative NS's at a false discovery rate < 0.01 . The $\mathrm{n}$ value, which varied from 5 to 10 , was proportional to the scaling level used in wavelet transform which in turn relate to the gene/probe density of the microarray platform. A high scaling level of wavelet transform increases the NS magnitude of neighbor points around a single differentiated gene, and thus makes them become statistically significant, which might in turn falsely identify a region as ICNV if $n$ is small (Additional File 1, Figure S1B). Therefore, $\mathrm{n}$ value is determined by the scaling level used in wavelet transform. The higher scaling level requires large $\mathrm{n}$. We have tested different $\mathrm{n}$ values from 5 to 10 for a given $\mathrm{s}=5$ in the Stanford data which is comprised of both aCGH and expression data. We have found that $\mathrm{n}=10$ provides the best overlap between aCGH-and expressionbased expression. Similarly, $\mathrm{n}=5$ which corresponds to $\mathrm{s}=3$, provides robust results for identifying recurrent ICNV regions. Finally, ICNV regions in multiple datasets were aligned to determine the recurrent $\mathrm{CNV}$ regions. The selection of filter function and scaling level for wavelet transform, as well as the validation of the method, is discussed in detail in Additional File 1.

Distinguishing cancer driver genes from passenger genes via reconstruction of Bayesian networks

$\mathrm{CNV}$ regions harbor many genes but only a small portion of them are cancer causal genes. A key challenge in cancer genomics is to distinguish the cancer driver genes, which are causal for oncogenesis and whose variations confer growth advantage on cancer cells, from the passenger genes that are physically located close to the driver genes in CNV regions[17]. Several methods have been proposed to indirectly identify driver genes by intersecting genes associated with $\mathrm{CNV}$ regions and gene coexpression networks[18] or protein-protein 
interaction data[19]. In our study, gene expression of two neighbor genes in an inferred $\mathrm{CNV}$ region (shown in Figure 3) are correlated to the inferred CNV (as implicated by the WACE method). To distinguish the potential cancer drivers, we tested whether they can causally regulate other genes instead. As shown in Figure 3 , two candidate genes $C_{1}$ and $C_{2}$ are cis regulated by a common CNV. To determine whether a downstream gene $\mathrm{G}$ is regulated by $\mathrm{C}_{1}$ or/and $\mathrm{C}_{2}$ or neither is equivalent to select among competing models:

$$
\begin{aligned}
& g \sim \varepsilon \\
& g \sim f\left(c_{1}\right) \\
& g \sim f\left(c_{2}\right) \\
& g \sim f\left(c_{1}, c_{2}\right)
\end{aligned}
$$

where eq. (1) represents $G$ independent of $C_{1}$ and $C_{2}$, eq.s (2) - (4) represent $G$ regulated by $C_{1}, C_{2}$, both $C_{1}$ and $\mathrm{C}_{2}$, respectively. As there are many other potential genes or factors that can affect the expression levels of gene G, the models eq. (1) - (4) are needed to condition on all other such factors. Thus, the model selection process is equivalent to a Bayesian network reconstruction process, which will be discussed subsequently.

Bayesian network is a probabilistic representation of the gene regulatory network and has shown superior performance in integrating genetic data into gene causal

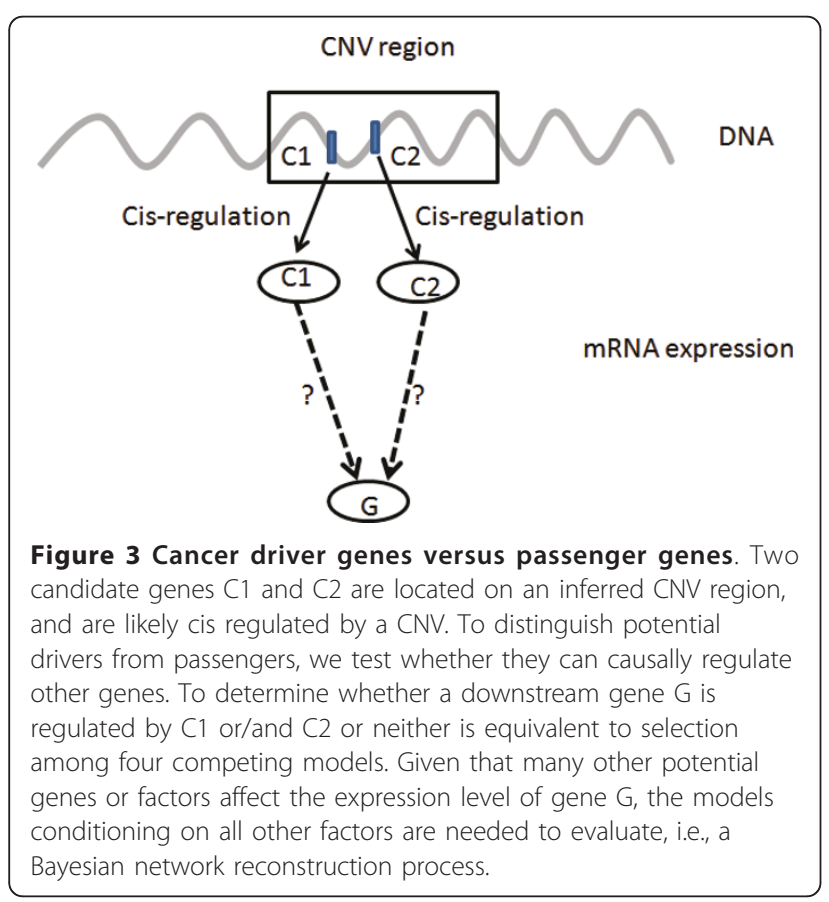

networks predictive of complex phenotypes[20-25]. 6,312, 6,349, 6,268 and 5,802 differentially regulated genes for NKI[14], Wang[6], Miller[15], and Christos[8], respectively, were selected as input into a Bayesian network reconstruction software program based on a previously described algorithm[24]. Markov Chain Monte Carlo (MCMC) simulation was then employed to identify the most plausible structures. For each seed, $15^{*} \mathrm{n}^{2}$ iterations of MCMC were run on average, where $n$ is the number of nodes. The Bayesian Information Criterion (BIC) scores were used as the optimization criteria. One thousand Bayesian networks were reconstructed using different random seeds to start the reconstruction process. From the resulting set of 1000 networks generated by this process, edges that appeared in greater than $30 \%$ of the networks were used to define a consensus network. The cutoff of $30 \%$ was based on a simulation study[26]. When summarizing 1000 constructed structures from random seeds, the histogram of the number of link occurrences among 1000 structures shows a bimodal distribution, where $30 \%$ is the best value to separate the two modes and also has the best recall-precision tradeoff [26]. Edges in this consensus network were removed if 1) the edge was involved in a loop, and 2) the edge was the most weakly supported of all edges making up the loop.

\section{Key Driver Analysis (KDA)}

One primary goal of gene network analysis is to identify key regulatory components, or key drivers, of sub-networks with respect to varying biological contexts $[25,27]$. The KDA takes as input a set of genes $(\mathbf{G})$ and a gene causal (directed) network $\mathbf{N}$. The objective is to identify the key regulators for the gene sets with respect to the given network. Candidate drivers are identified as follows. We first compute the size of the h-layer neighborhood (HLN) for each node. The range of $h$ is from 1 to the diameter of the network N. Specifically, for a given node $g$, the size of its HLN is the number of its downstream nodes that are within h edges away from $g$. For the given network $\mathbf{N}$, let $\boldsymbol{\mu}$ be an array of the sizes of HLNs and $\boldsymbol{d}$ be an array of the out-degrees for all the nodes. The nodes are nominated as candidate drivers if their sizes of their HLN are greater than $\bar{\mu}+\sigma(\mu)$, where $\bar{\mu}$ is the mean of $\boldsymbol{\mu}$ is and $\sigma(\mu)$ is the standard deviation of $\mu$. The candidate drivers without any parent node (i.e., root nodes) are nominated as global drivers while the rest are local regulators. Let $\bar{d}$ be the mean of $\boldsymbol{d}$ and $\sigma(d)$ be the standard deviation of $\boldsymbol{d}$. We also promote hub nodes as global drivers, i.e., the nodes with out-degrees above $\bar{d}+2 \sigma(d)$ are designated as global drivers. These criteria identify genes with number of downstream nodes or number of outlinks significantly above the average. 
By definition, each driver modulates a set of genes, i.e., its downstream nodes. Previous work has shown that a gene's function can be predicted by its neighbor genes in networks[28]. Moreover, a series of validation experiments show that the downstream nodes of a driver predicted by Bayesian networks significantly overlap with its knockout signature[25].

\section{siRNA Screen and Cell Viability Assays Cell lines and siRNA library}

Four different breast cancer cell lines, ER-positive MCF7 and ZR-75-1; and ER-negative MDA-MB-231and MDAMB-468[29], were obtained from the American Type Culture Collection, Rockville, MD (Catalogue numbers HTB-22, HTB-26, HTB-132 and CRL-1500, respectively). The siRNA library targets $\sim 2,400$ unique human genes, with three siRNAs per gene, as described previously[30]. The library represents genes comprising kinases, membrane proteins, enzymes, components of major cellular pathways including cell cycle, transcription regulation, and signal transduction, etc. [31]. siRNA sequences were designed with an algorithm developed to increase efficiency of the siRNAs for silencing while minimizing their off-target effects [32]. siRNAs were ordered from Sigma-Proligo (The Woodlands, TX).

\section{siRNA screen and cell viability assays}

siRNA screens were performed as described previously [30] and cells were transfected using RNAiMAX (Invitrogen, Carlsbad, CA). Cell viability assay was determined using AlamarBlue reagent (BioSource International, Camarillo, CA). The fluorescence signal was corrected for background (no cells). Cell growth was expressed as \% viability relative to the median value of wells transfected with an siRNA to Luciferase.

\section{Results}

As detailed in the Materials and Methods, the proposed unified framework for expression based CNV inference and causal gene identification is comprised of three major components, wavelet based CNV inference, causal network construction and key driver identification.

\section{Performance Comparison of WACE and GACE}

The original ACE approach for identifying amplified or deleted chromosome regions used the simple Gaussian transform to smooth the data and then identified the significantly abnormal regions comprised of over- or under-expressed genes via a permutation test[13]. Although this approach, named GACE, was able to narrow down genes whose expression might be affected by the local CNV, it often systematically overestimated the size of the identified regions which were typically rearrangements of small sequences. We improved GACE by introducing: i) a wavelet based smoothing technique and ii) a new statistical method for assessing significance of putative $\mathrm{CNV}$ regions. Wavelet transform is a more sophisticated filtering technique and has become a cutting-edge technology in signal and image processing because of its superior ability to accurately deconstruct and reconstruct finite, non-periodic and/or non-stationary signals[33]. This led to a wavelet-based ACE algorithm (WACE) to efficiently and accurately narrow down amplified/deleted regions of the genome in cancer samples.

To access the performance of WACE, first we compared it with the existing method GACE based on a previously published breast cancer study [16], which consisted of gene expression and aCGH data, as well as clinical data relating to tumor progression (referred to here as BCS1). A detailed comparison is described in Additional File 1, WACE and GACE Comparison (Section 2.1). Figure S4 to $S 6$ in Additional File 1 illustrated the comparison results between two methods based on (i) the correlation coefficient between the expressionand aCGH-based NS profiles, and (ii) re-identifying CNVs based on gene expression, respectively. Here we summarize the findings: (i) WACE uncovered almost three times as many expression ICNV regions overlapping with the aCGH ICNV regions compared to GACE, and (ii) these two sets of regions identified by WACE were better correlated with each other than those identified by GACE.

We also verified the cis-effect of CNV on gene expression by considering the correlation between the aCGH and gene expression data of each gene in BCS1. The expression of the genes located in the overlapped CNV regions was highly correlated to the corresponding aCGH data. For instance, WACE identified in both expression and aCGH data a DNA amplification region at $20 \mathrm{q} 13$, and the gene expression and aCGH NS profiles were highly correlated $(\mathrm{r}=0.55)$.

\section{Amplified regions associated with poor outcome affect cell cycle}

We applied WACE to the aforementioned four breast cancer microarray studies $[6,8,14,15]$ to infer CNV regions associated with metastasis. Each dataset was independently analyzed by WACE and the identified regions were then aligned to locate the recurrent regions of ICNVs. To calculate the ES's for each dataset, the samples were classified into two groups, the patients with metastases within 5 years and those with no metastasis after more than 5 years of follow-up.

Figure 4 shows the NS profiles on chromosome 8. WACE identified the amplified chromosome 8q21-q23 region (around $100 \mathrm{Mb}$ and harboring the $M T D H$ gene) in three of the four datasets. The amplification of this region has previously been experimentally verified [13]. 


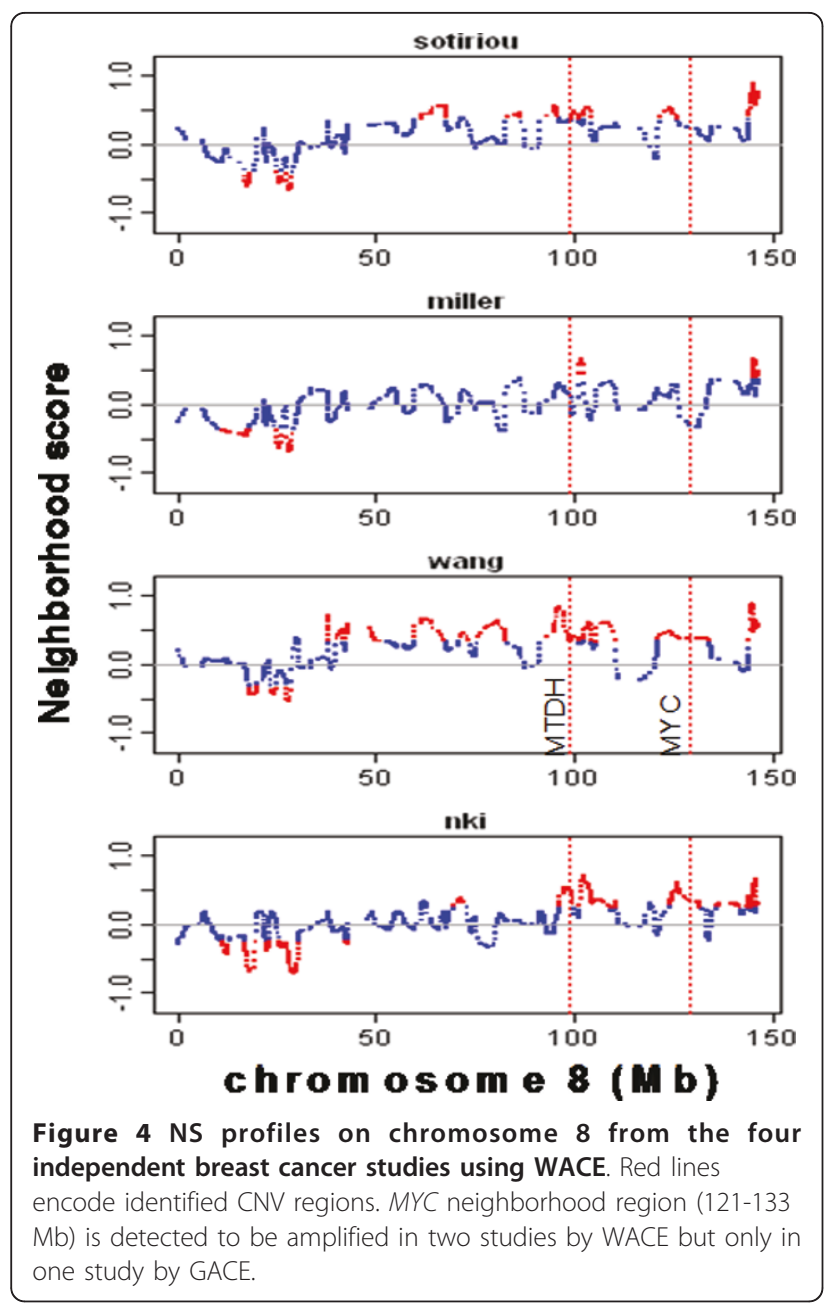

It also identified CNV in the 8p21-p12 and 8q24.3 cytobands associated with tumor outcome. The $8 \mathrm{q} 24$ cytoband, which was consistently detected as an amplified region by WACE, was found to be the most frequently amplified region by genome-wide array $\mathrm{CGH}[9,12]$, where the expression of the underlying genes also reflected the change in DNA copy number[12]. Moreover, this region also includes the well-known oncogene $M Y C$.

The recurrent regions of CNV were defined as the union of the regions in which the abnormal gain/loss events were identified in at least two out of the four studies. WACE found 109 recurrent regions covering 2,560 genes (Table 1 and Additional File 1, Table S1). Table 1 lists the recurrent regions of amplification that were consistently found by WACE in all four datasets. Several regions on chromosome 8,11 , and 20 identified here have been previously identified via analysis of CGH arrays applied to breast cancer tumors [10]. These results further confirm the accuracy of WACE. We note that many of the genes in the inferred recurrent regions were oncogenes (19 of total 84 oncogenes according to Panther database) or significantly affected the cell viability based on siRNA experiments (Figure 5 ).

These regions were tested for gene enrichment using the gene ontology (GO) categories, KEGG, Panther, and GeneGo pathways. The most enriched categories include nucleosome assembly $(\mathrm{P}<7.24 \mathrm{E}-22)$, chromatin assembly $(\mathrm{P}<1.02 \mathrm{E}-20)$, systemic lupus erythematosus $(\mathrm{P}<$ 9.03E-18), chromatin assembly or disassembly $(\mathrm{P}<$ 4.57E-17), DNA packaging $(\mathrm{P}<1.29 \mathrm{E}-16)$ and proteinDNA complex assembly $(\mathrm{P}<1.92 \mathrm{E}-11)$. Cell cycle and cholesterol biosynthetic process pathways are also enriched in the regions. All the enriched categories are shown Additional File 1, Table S3.

\section{ICNV regions versus aCGH based regions}

We further examined to what extent the recurrent CNVs inferred by gene expression reflect the recurrent CNVs based on an independent aCGH data in BCS1. To ensure an objective comparison, we used a popular CNV detection method, the circular binary segmentation (CBS) algorithm (implemented in the DNAcopy package from Bioconductor) on the aCGH data. Forty six or $68.7 \%$ of the $67 \mathrm{CBS}$ based aCGH CNV regions in BCS1 significantly (gene-based FET $\mathrm{P}=3.1 \mathrm{e}-13$ ) overlapped with the 109 recurrent ICNV regions detected by WACE on the four gene expression studies. The overlapped regions on chromosome 8,11 , and 20 have been previously identified via analysis of CGH arrays applied to breast cancer tumors [10]. These results demonstrate a good correspondence between the inferred CNV regions and the experimental results and thus confirm the accuracy of WACE.

\section{Breast Cancer Gene Regulatory Networks}

Four whole-genome gene regulatory networks were first constructed by the Bayesian network reconstruction method [26] for the aforementioned four breast cancer studies (NKI, Christos, Miller, and Wang) and then were combined by union of directed links to form a single network, which consisted of 10,118 genes and 20,732 directed links. Previous works have shown that genes controlling many other genes in gene regulatory networks are more likely regulatory genes regulating their downstream genes[24,25,27]. The top 5 genes with the most out links are ARF1, FOXA1, ESR1, HIF1A and $U B E 2 C$. ARF1 is involved in the activation of the PI3K/ AKT pathways which regulate cell survival and proliferation[34]. HIF1A plays an essential role in cellular and systemic homeostatic response to hypoxia was recently found to regulate the metastasis of breast tumor to lung [35]. FOXA1, ESR1 and UBE2C, are known as breast cancer prognosis biomarkers[36]. The global features of the combined network are described in detail in 
Table 1 The inferred recurrent regions of CNV in the four breast cancer datasets.

\begin{tabular}{|c|c|c|c|c|c|c|}
\hline $\begin{array}{l}\text { Cytoband } \\
\text { Location }\end{array}$ & Chr & $\begin{array}{l}\text { Start } \\
(\mathrm{Mb})\end{array}$ & $\begin{array}{l}\text { End } \\
(\mathrm{Mb})\end{array}$ & $\begin{array}{l}\text { Size } \\
(\mathrm{Mb})\end{array}$ & $\begin{array}{l}\text { Gain/ } \\
\text { Loss }\end{array}$ & Important genes \\
\hline chr3q26.31-q27.1 & 3 & 173.95 & 185.91 & 11.96 & G & ECT2, PIK3CA, PSMD2, CLCN2, POLR2H \\
\hline chr4p16.3 & 4 & 0.69 & 1.95 & 1.26 & G & CTBP1, TACC3 \\
\hline chr5q35.2-q35.4 & 5 & 173.41 & 179.09 & 5.69 & G & GRK6, DDX41 \\
\hline chr8p21.2-p12 & 8 & 23.59 & 30.70 & 7.11 & $\mathrm{~L}$ & CLU \\
\hline chr8q24.3 & 8 & 143.54 & 146.25 & 2.71 & G & NRBP2 \\
\hline chr11q13.1-q13.4 & 11 & 64.54 & 71.59 & 7.05 & G & MAP3K11, ADRBK1, RPS6KB2, LRP5, CPT1A \\
\hline $\begin{array}{l}\text { chr12q24.23- } \\
\text { q24.33 }\end{array}$ & 12 & 120.70 & 122.32 & 1.62 & G & \\
\hline chr16p13.4 & 16 & 2.02 & 2.66 & 0.64 & G & DCl, PDPK1 \\
\hline chr16q12.2-q22.1 & 16 & 54.25 & 69.11 & 14.87 & G & POLR2C, KIFC3, CSNK2A2, GOT2, CDH5, PSKH1, PSMB10, VPS4A, \\
\hline chr16q22.3-q24.3 & 16 & 82.56 & 88.62 & 6.06 & G & GALNS \\
\hline chr20q11.1-q11.23 & 20 & 28.24 & 36.99 & 8.75 & G & $\begin{array}{l}\text { TPX2, APBA2BP, E2F1, EIF2S2, AHCY, GSS, PROCR, RBM39, SCAND1, DLGAP4, } \\
\text { GHRH, BLCAP }\end{array}$ \\
\hline $\begin{array}{l}\text { chr20q13.12- } \\
\text { q13.13 }\end{array}$ & 20 & 44.43 & 49.01 & 4.58 & G & ARFGEF2, CSE1L \\
\hline
\end{tabular}

The important genes include known oncogenes, known tumor suppressors, and the genes that, when silenced, reduced cell viability by more than $50 \%$ based on our siRNA experiments.

Additional File 1, Properties of Breast Cancer Bayesian Networks (Section 3).

\section{Key Driver Analysis}

We assume that the cancer driver genes have broad impacts on global gene expression while passenger genes have small impacts [37]. To quantify impacts of genes residing in the recurrent ICNVs detected across multiple data sets, we count numbers of downstream genes that are regulated by each candidate gene locally or globally in the combined network. Genes are grouped into global drivers, local drivers and passengers according to the rank of these numbers (see Methods for details). In the key driver analysis, we focus on the genes residing in amplified regions which are regarded as a hallmark of dominant cancer driver genes[17].

For the genes in the recurrent regions of amplification (RR Gain), we identified 44 global drivers and 24 local drivers. Known breast cancer susceptibility genes like TPX2, AURKA, TK1 and BIRC5 are among the top 7 global drivers (the other 3 top drivers are GINS2, COX4NB and NUP93). Other known breast cancer gene target such as ERBB2, E2F1 and MTDH are in the global driver list. Many of them such as aurora kinases $A U R K A$ and survivin (BIRC5) have been targets for anticancer drugs [38-40].

To access the robustness of predicted key drivers, we performed the KDA on the networks (named as the $3 \mathrm{~N}$ networks) as combinations of any three of the four Bayesian networks. Over $79 \%$ of the drivers based on the $3 \mathrm{~N}$ networks are the drivers based on the combination of all the four BNs. On the other hand, the four driver sets based on the $3 \mathrm{~N}$ networks significantly overlap with each other (with FET p-values $<1 \mathrm{E}-14$ ) and they share at least $41 \%$ of their members. Therefore, the drivers uncovered are fairly robust. It is of note that the network based on all the four networks leads to many more drivers than any $3 \mathrm{~N}$ network, indicating the great advantage of the combination of all the networks.

\section{Validation of key drivers via in vitro siRNA knockdown experiments}

We assessed the effect of knocking down the genes located on the amplified recurrent ICNV regions by siRNA on breast cancer cell viability because they were enriched for gene sets involved in cell cycle and metabolic regulation. In the previously described siRNA experiments, the median of the viability of the siRNA transfected cells was $64.30 \%$, with a standard deviation of $15.28 \%$, compared to the control cells transfected with an siRNA to luciferase. We used a viability cutoff value of $41.38 \%$ ( $1.5 \times$ standard deviation below the median) to determine viability signature genes that, when silenced, significantly decrease cell viability. This resulted in the identification of a 216 gene signature (V1) with a significant effect on cell viability in multiple cell lines. Based on a cutoff of $1.5 \times$ standard deviation below the median, we also derived a signature for each cell line. The four signatures from individual cell lines were then combined with V1 to derive a combined signature V2 with 484 genes. The genes in V1 and V2 and their viability scores are shown in Additional File 2, Table S4. 
We assessed whether V1 and V2 signatures are significantly enriched in different gene sets including the global drivers, the local drivers, the drivers (global and local), the non-driver genes and the genes not on the recurrent regions (nonRR). Figure 5 shows the fold enrichment of each gene set for the siRNA hit signatures V1 and V2. When compared with the genes not on the recurrent ICNV regions, the genes on these regions are about twice (i.e. 2.1 and 1.8 vs. 0.9 ) more likely to fall into V1 and V2, respectively. Also the global drivers are most likely to be siRNA hits in both V1 and V2, followed by local drivers and non-drivers. For example, the global drivers, the local drivers and the non drivers are $7.8(\mathrm{P}<5.8 \mathrm{e}-4), 4.8(\mathrm{P}<1.9 \mathrm{e}-2)$ and $1.97(\mathrm{P}<2.5 \mathrm{e}-6)$ times more likely to overlap with $\mathrm{V} 1$, respectively than the genes on the human genome. When the genes on the recurrent ICNV regions were considered as background, the global drivers were 4.2 ( $\mathrm{P}$ $<0.0055)$ and $3.5(\mathrm{P}<0.003)$ times more likely to be in V1 and V2, respectively, and the local drivers are less significantly enriched for the signatures.

Seven drivers (ATP6V1C1, BIRC5, DDX19A, DDX28, E2F1, PSMC5 and TPX2) are in V1. The protein Survivin is encoded by BIRC5 (located in 17q25) and is wellknown for its role in mitotic regulation and apoptosis, and has been developed as a target for cancer treatment

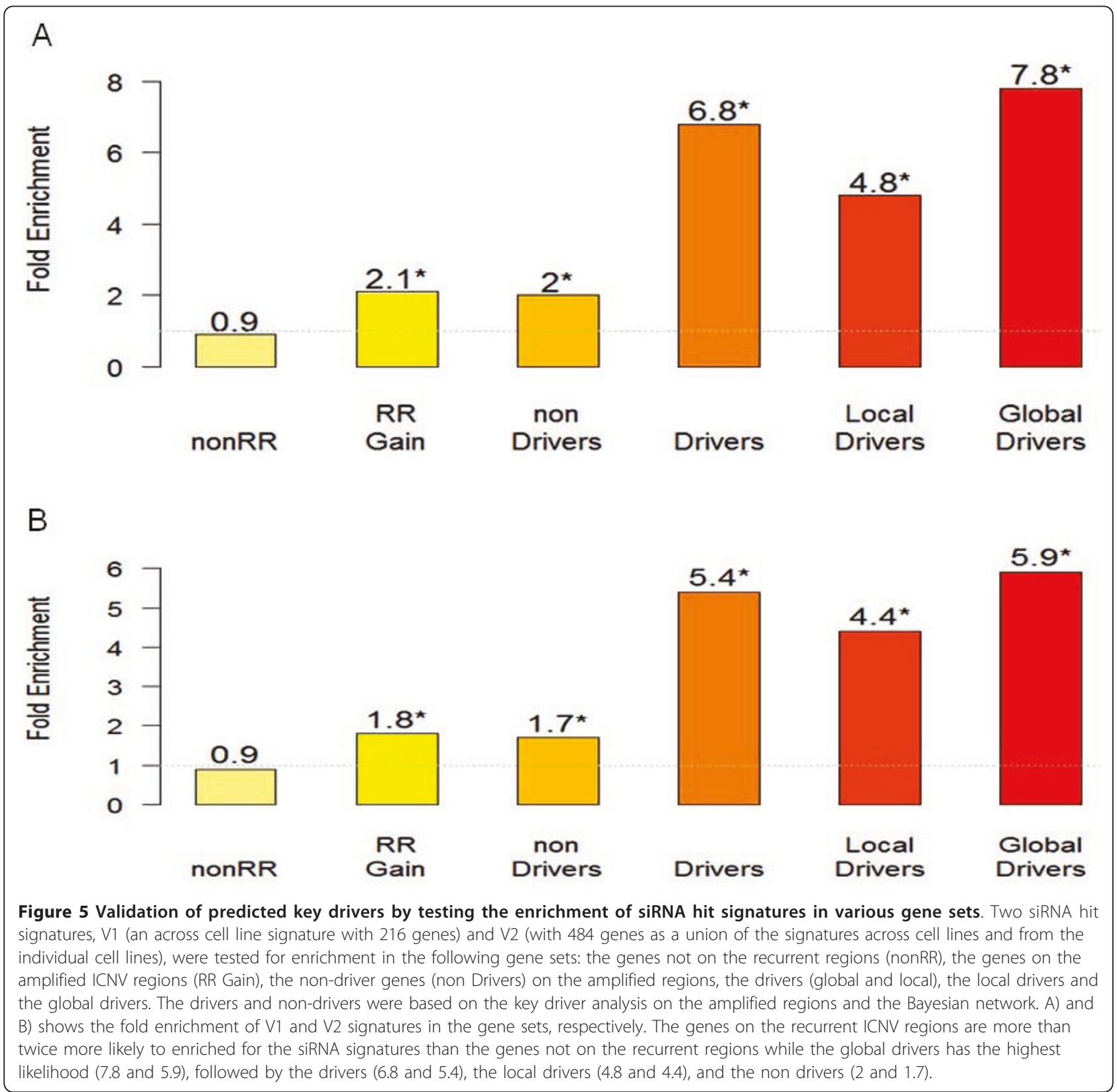


[39]. Silencing BIRC5 decreased the cellular viability by $64 \%$. TPX2 is located on $20 \mathrm{q} 11$ and the cell viability dropped by $64 \%$ when it was silenced. Figure 6 shows the subnetwork associated with the amplified recurrent ICNV regions. The global and local drivers are highlighted in different sizes. The genes found in the signature V1 are highlighted in green.

As mentioned earlier, AURKA and TPX2 are the top key regulators of the recurrent ICNV regions. On the other hand, 107 of the genes on the recurrent regions overlapped with $\mathrm{V} 2$ and they include TACC3, ECT2, CDC6, NOTCH1, and PIK3CA etc. Here we found a molecular mechanism that linked TACC 3 and TPX2 through AURKA. TACC3, located on an amplified region on $4 \mathrm{p} 16$, recently emerged as an important gene in the stabilization of the mitotic spindle[41], and is upregulated in several cancer cell lines including thyroid and lung cancers[42-44]. Cell viability was reduced by $59.80 \%$ in the TACC3 siRNA transfected cells. AURKA resides on 20q13, which was also identified as a recurrent region of amplification. It has previously been shown that TPX2 is one of several activators for AURKA [45], which in turn controls the localization of TACC3 to the spindles [46]. Furthermore, TPX2 acts as an allosteric activator of Aurora A, and together they inhibit the activity of tumor suppressor p53 in Xenopus Oocytes (Pascreau et al. 2009). The molecular interaction between the hubs located on different recurrent regions might suggest the trans-association between regions. We expect more mechanisms of this kind will be unraveled through the integration of WACE and network analysis.

These experimental results demonstrate the importance of the recurrent ICNV regions and the predicted key driver genes, especially global drivers in terms of significant impact on cancer cell viability, and thus validate our procedures for inferring $\mathrm{CNV}$ regions and identifying cancer driver genes in these inferred $\mathrm{CNV}$ regions.

\section{Discussion}

We developed a novel Wavelet based algorithm to Analyze Copy number alteration based on Expression (WACE) and further combined this analysis with gene regulatory network analysis to identify the cancer driver genes in the chromosome regions with genomic alterations. The performance of these two methods was evaluated in Additional File 1, Section 2. In brief, WACE improved on the state-of-the-art algorithm (GACE) by

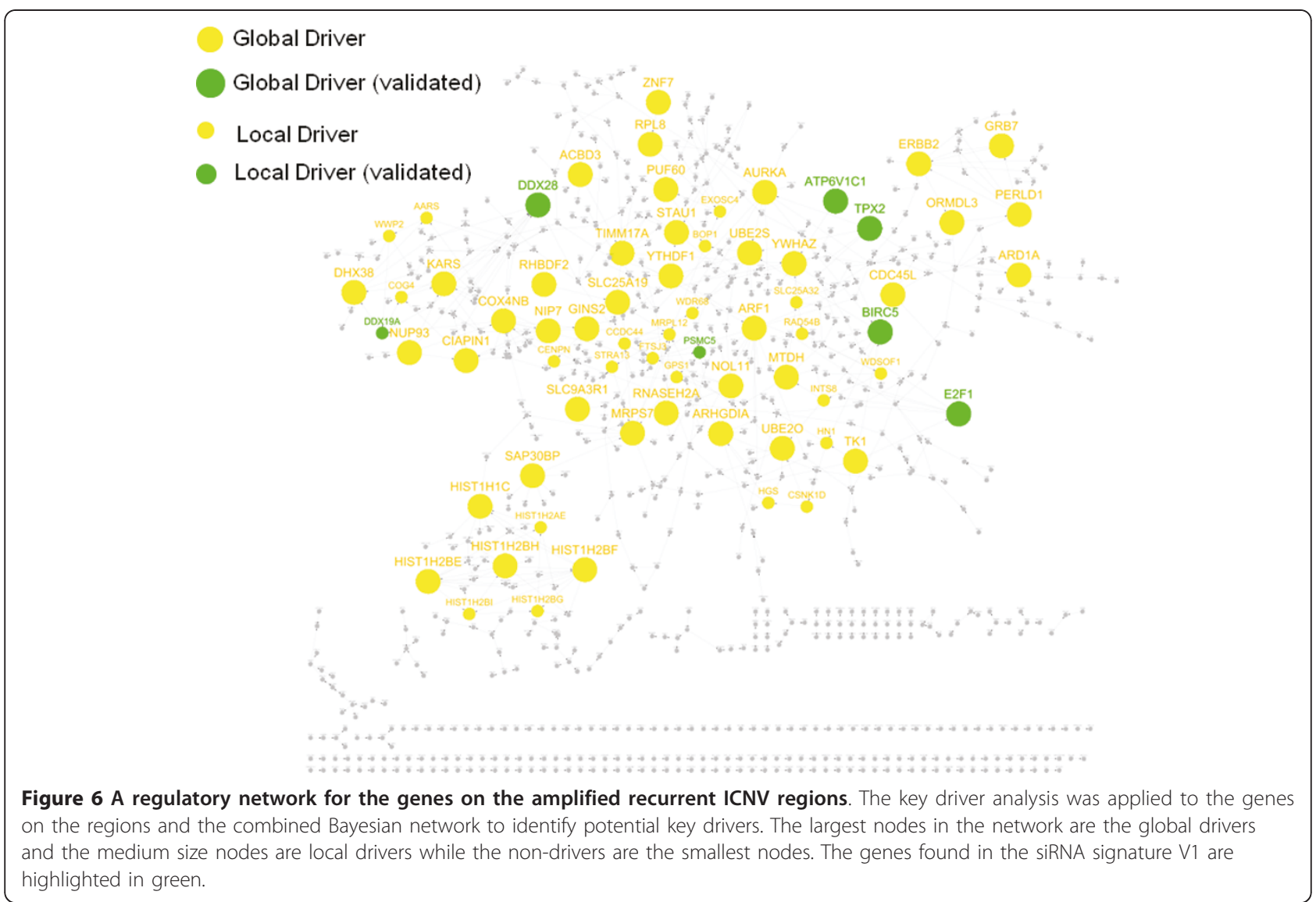


(i) reducing the over-smoothing effect of the Gaussian transform and retaining the local patterns, and (ii) eliminating the type I and II errors induced by the non-zero centered null distribution (Additional File 1, Figure S2). WACE demonstrated superior performance over GACE on several large scale gene expression datasets (see Additional File 1, Section 2). By analyzing multiple datasets, we were able to identify recurrent ICNVs that harbor many cancer-related genes, suggesting genes in these regions are worthy of further study.

Integration of the recurrent regions identified by WACE and the gene regulatory network analysis uncovered not only many well-known oncogenes like BIR$C 5$ and $E 2 F 1$, but also a number of novel cancer susceptibility genes such as ECT2, TPX2 and TACC3, which are involved in cell cycle and ECM regulation. Many of the cancer driver genes we predicted were subsequently validated via siRNA experiments. Table S5 in Additional File 1 includes the four known and 45 novel breast cancer genes that are in the recurrent regions of amplification and validated by the siRNA experiments across multiple breast cancer cell lines.

The importance of the identified recurrent regions of amplification (ARR) and the inferred drivers are further justified by another independent dataset from Cancer Gene Census http://www.sanger.ac.uk/genetics/CGP/ Census/. Among the 19 known breast cancer genes compiled by Cancer Gene Census, 5 are located in the ARRs while 14 are outside the ARRs, indicating that the known breast cancer driver genes are 3.5-fold overrepresented in the ARRs when compared to the genes not on these regions. Among the 5 census genes located in the ARRs, one (ERBB2) is the global key driver and the rest four are not the drivers, representing 25- and 2-fold enrichments, respectively, compared to randomly selected human genes.

There are multiple molecular factors contributing to poor outcome, including cell proliferation, metastasis and cell stemness etc. Our assumption is that CNV significantly contributes to the disease outcome though the separation of the samples into good and bad outcome groups tends to identify metastasis genes. We don't need to pre-specify through what molecular mechanism that CNV drives disease outcome, which can be inferred in a data-driven manner. To further understand the functions of the ARRs, we performed gene co-expression network analysis $[47,48]$ on the four breast cancer datasets to identify gene modules (comprised of highly interacted genes). A module enriched for cell cycle genes (named as cell cycle module) and conserved across the four corresponding networks is most predictive of survival in all the four studies, indicating cell proliferation contributes to breast cancer outcome. The ARRs are highly enriched for the genes of the cell cycle module $(\mathrm{P}<1.2 \mathrm{e}-14)$. This is further supported by the fact that the inferred key drivers include not only known breast cancer metastasis genes (MTDH[13] and $E R B B 2[49])$ but also known cancer cell cycle genes such as TPX2, AURKA, E2F1 and BIRC5. As shown in Figure 6 , these known cell cycle and metastasis genes interact with each other and with many other drivers to co-regulate the genes on the AARs. Furthermore, AARs are enriched (2.3 fold-enrichment, $\mathrm{P}<2.6 \mathrm{e}-2$ ) for a cell cycle prognostic signature identified as metastasis markers[50], and two (AURKA and BIRC5) of the thirtythree signature genes are the global key drivers (32 foldenrichment, $\mathrm{P}<1.8 \mathrm{e}-3)$. Therefore, cell viability assay, used to test cell proliferation, at least partially validated our drivers. The future experiments will consider cell mobility assay to test how these inferred key drivers impact cancer cell mobility that drives metastasis.

A recent paper by Akavia et al. describes a method to predict cancer driver genes by integrating $\mathrm{CNA}$ and gene expression data[51]. Akavia et al's approach bears certain similarity as ours. Both approaches first define gene groups of interest, then identify genes in the groups with cis-CNV as candidate driver genes. As we discussed in the main text, expression levels of genes in the same CNV segments are likely to be correlated so that they are assigned into the group. Candidate genes (with cis-CNV) in the same co-expression module are equivalent so that we cannot rank which candidate genes are more likely to be drivers. Akavia et al. used a literature based method to rank candidate genes. Basically, they chose genes with known connections to cancers. However, our approach is completely data driven. We first constructed causal networks to dissect how genes in the regions of interest are regulated and related to each other and then we ranked candidate genes based on the causal networks, which is the key driver analysis described in the main text. As we mentioned in the previous discussion, majority of the drivers identified by our approach are novel and thus they won't be picked up by Akavia et al's literature based approach.

Jornsten et al. recently developed the EPoC method to integrate CNA and gene expression variations[52]. EPoC focuses on CNA driving gene expression changes but disregards the indirect changes through more hubs. Only genes with CNA can be cancer driver genes based on the EPoC model. However, our Bayesian network approach focuses on how genes are causally related to each other. In our case, predicted cancer driver genes may or may not have copy number alterations. Theoretically, the EPoC method can also derive gene-gene relationship as Jorsten et al. indicated that gene-gene relationship matrix $A$ is the inversion of the CNA-gene relationship matrix $\boldsymbol{G}\left(\boldsymbol{G}=\boldsymbol{A}^{-1}\right)$. However, they showed that there was no prediction power using the $\mathbf{A}$ matrix. 
One reason is that in their Lesso formulation for $\boldsymbol{G}$ matrix, they explicitly assumed CNAs are hubs so that $\boldsymbol{G}^{-1}$ is not exactly $\boldsymbol{A}$. We have showed that gene-gene networks based on our Bayesian network approach have prediction power[24-27]. Thus, our method can be complimentary to the EPoC method.

The impact of CNV on gene expression has been extensively studied[53,54]. In our analysis, we focused on genes with cis-regulated CNVs. CNVs may result partial deletions of genes and the functional changes of the affected genes in turn cause expression changes of downstream genes. Gene regulatory networks may be able to capture the effect of these cis-regulated CNVs on the trans-regulated ones. In our model, we explicitly assume CNVs cause gene expression changes. There are many mechanisms that CNV can be arisen[55]. It is possible that expression changes of genes in DNA repair processes affect CNV. More sophisticated causal models, such as dynamic Bayesian network, are needed to capture these causal relationships. It is of note that there is a limitation of this transcriptome-based approach. We may miss kinases or enzymes that drive cancer progression and metastasis if these kinases' or enzymes' activity changes are mainly due to protein level changes. Complementary proteomic approaches are needed to complement this approach.

\section{Conclusion}

The framework where the wavelet analysis of copy number alteration based on expression coupled with the gene regulatory network analysis, provides a blueprint for leveraging genomic data to identify key regulatory components and gene targets. To our knowledge, this is the first effort to systematically identify and validate drivers for expression based CNV regions in breast cancer. This integrative approach can be applied to many other large-scale gene expression studies and other novel types of cancer data such as next-generation sequencing based expression (RNA-Seq) as well as CNV data.

\section{Additional material}

Additional file 1: Supplementary Information. Supplementary

methods and results.

Additional file 2: Supplementary Table S4. siRNA screen signatures and the associated viability scores.

\section{Acknowledgements}

This study was funded in part by Merck \& Co.

\section{Author details}

'Sage Bionetworks, Seattle, WA 98109, USA. ${ }^{2}$ Merck Research Laboratories, Merck \& Co., Inc., 33 Avenue Louis Pasteur, Boston, MA 02115, USA. ${ }^{3}$ Pacific Biosciences, 1505 Adams Drive, Menlo Park, California 94025, USA.

\section{Authors' contributions}

$B Z, J Z$ and ES conceived and designed the experiments. LT and BZ designed the data analysis scheme. CZ, TX, JL, HD and JZ collected samples and preprocessed the data. $L T, B Z, Z Z, C Z, T X, J L$ and $J Z$ analyzed the data. ZZ performed validation experiments. $L T, B Z, Z Z, J Z$ and ES wrote the paper. BZ coordinated the study. All the authors read and approved the final manuscript.

\section{Competing interests}

The authors declare that they have no competing interests.

Received: 14 March 2011 Accepted: 1 August 2011

Published: 1 August 2011

\section{References}

1. Hebbring SJ, Moyer AM, Weinshilboum RM: Sulfotransferase gene copy number variation: pharmacogenetics and function. Cytogenet Genome Res 2008, 123(1-4):205-10.

2. Mullighan CG, Downing JR: Genome-wide profiling of genetic alterations in acute lymphoblastic leukemia: recent insights and future directions. Leukemia 2009.

3. Overdevest JB, Theodorescu D, Lee JK: Utilizing the molecular gateway: the path to personalized cancer management. Clin Chem 2009, 55(4):684-971.

4. Swanton C, Caldas C: Molecular classification of solid tumours: towards pathway-driven therapeutics. Br J Cancer 2009.

5. van 't Veer $L$, et al: Gene expression profiling predicts clinical outcome of breast cancer. Nature 2002, 415(6871):530-6.

6. Wang $Y$, et al: Gene-expression profiles to predict distant metastasis of lymph-node-negative primary breast cancer. Lancet 2005, 365(9460):671-9.

7. Fan C, et al: Concordance among gene-expression-based predictors for breast cancer. N Engl J Med 2006, 355(6):560-9.

8. Sotiriou $C$, et al: Gene expression profiling in breast cancer: understanding the molecular basis of histologic grade to improve prognosis. J Natl Cancer Inst 2006, 98(4):262-72.

9. Andre F, et al: Molecular characterization of breast cancer with highresolution oligonucleotide comparative genomic hybridization array. Clin Cancer Res 2009, 15(2):441-51.

10. Haverty PM, et al: High-resolution genomic and expression analyses of copy number alterations in breast tumors. Genes Chromosomes Cancer 2008, 47(6):530-42

11. Heidenblad $M$, et al: Microarray analyses reveal strong influence of DNA copy number alterations on the transcriptional patterns in pancreatic cancer: implications for the interpretation of genomic amplifications. Oncogene 2005, 24(10):1794-801.

12. Pollack JR, et al: Microarray analysis reveals a major direct role of DNA copy number alteration in the transcriptional program of human breast tumors. Proc Natl Acad Sci USA 2002, 99(20):12963-8.

13. $\mathrm{Hu} \mathrm{G}$, et al: $\mathrm{MTDH}$ activation by $8 \mathrm{q} 22$ genomic gain promotes chemoresistance and metastasis of poor-prognosis breast cancer. Cancer Cell 2009, 15(1):9-20.

14. van 't Veer $L$, et al: Gene expression profiling predicts clinical outcome of breast cancer. Nature 2002, 415(6871):530-6.

15. Miller $L D$, et al: An expression signature for $p 53$ status in human breast cancer predicts mutation status, transcriptional effects, and patient survival. Proc Natl Acad Sci USA 2005, 102(38):13550-5.

16. Bergamaschi $A$, et al: Distinct patterns of DNA copy number alteration are associated with different clinicopathological features and geneexpression subtypes of breast cancer. Genes Chromosomes Cancer 2006, 45(11):1033-40.

17. Stratton MR, Campbell PJ, Futreal PA: The cancer genome. Nature 2009, 458(7239):719-24.

18. Torkamani $A$, et al: Coexpression network analysis of neural tissue reveals perturbations in developmental processes in schizophrenia. Genome Res 2010, 20(4):403-12.

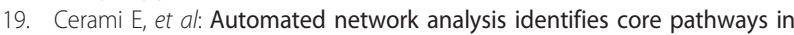
glioblastoma. PLOS ONE 2010, 5(2):e8918.

20. Kulp DC, Jagalur M: Causal inference of regulator-target pairs by gene mapping of expression phenotypes. BMC Genomics 2006, 7:125. 
21. Lum PY, et al: Elucidating the murine brain transcriptional network in a segregating mouse population to identify core functional modules for obesity and diabetes. J Neurochem 2006, 97(Suppl 1):50-62.

22. Mehrabian $M$, et al: Integrating genotypic and expression data in a segregating mouse population to identify 5-lipoxygenase as a susceptibility gene for obesity and bone traits. Nat Genet 2005, 37(11):1224-33.

23. Schadt EE, et al: An integrative genomics approach to infer causal associations between gene expression and disease. Nat Genet 2005 37(7):710-7.

24. Zhu J, et al: An integrative genomics approach to the reconstruction of gene networks in segregating populations. Cytogenet Genome Res 2004, 105(2-4):363-74.

25. Zhu J, et al: Integrating large-scale functional genomic data to dissect the complexity of yeast regulatory networks. Nat Genet 2008, 40(7):854-61.

26. Zhu J, et al: Increasing the Power to Detect Causal Associations by Combining Genotypic and Expression Data in Segregating Populations. PLoS Comput Biol 2007, 3(4):e69.

27. Yang $X$, et al: Systematic genetic and genomic analysis of cytochrome P450 enzyme activities in human liver. Genome Res 2010.

28. Sharan R, Ulitsky I, Shamir R: Network-based prediction of protein function. Mol Syst Biol 2007, 3:88.

29. Roodi $N$, et al: Estrogen receptor gene analysis in estrogen receptorpositive and receptor-negative primary breast cancer. J Natl Cancer Inst 1995, 87(6):446-51.

30. Bartz SR, et al: Small interfering RNA screens reveal enhanced cisplatin cytotoxicity in tumor cells having both BRCA network and TP53 disruptions. Mol Cell Biol 2006, 26(24):9377-86.

31. Berns $K$, et al: A large-scale RNAi screen in human cells identifies new components of the p53 pathway. Nature 2004, 428(6981):431-7.

32. Jackson $A L$, et al: Expression profiling reveals off-target gene regulation by RNAi. Nat Biotechnol 2003, 21(6):635-7.

33. Strang GN, Truong: Wavelets and Filter Banks. Wellesley, MA, USA: Wellesley-Cambrage Press; 1996.

34. Boulay PL, et al: ADP-ribosylation factor 1 controls the activation of the phosphatidylinositol 3-kinase pathway to regulate epidermal growth factor-dependent growth and migration of breast cancer cells. J Biol Chem 2008, 283(52):36425-34.

35. Lu $X$, et al: In vivo dynamics and distinct functions of hypoxia in primary tumor growth and organotropic metastasis of breast cancer. Cancer Res 2010, 70(10):3905-14.

36. Nakshatri H, Badve S: FOXA1 in breast cancer. Expert Rev Mol Med 2009 11:e8

37. Torkamani A, Schork NJ: Identification of rare cancer driver mutations by network reconstruction. Genome Res 2009, 19(9):1570-8.

38. Macarulla T, Ramos FJ, Tabernero J: Aurora kinase family: a new target for anticancer drug. Recent Pat Anticancer Drug Discov 2008, 3(2):114-22.

39. Mita AC, et al: Survivin: key regulator of mitosis and apoptosis and novel target for cancer therapeutics. Clin Cancer Res 2008, 14(16):5000-5.

40. Rana $S$, et al: Stathmin 1: a novel therapeutic target for anticancer activity. Expert Rev Anticancer Ther 2008, 8(9):1461-70.

41. Peset I, Vernos I: The TACC proteins: TACC-ling microtubule dynamics and centrosome function. Trends Cell Biol 2008, 18(8):379-88.

42. Fridlyand J, et al: Breast tumor copy number aberration phenotypes and genomic instability. BMC Cancer 2006, 6:96.

43. Still $I H$, Vince $P$, Cowell JK: The third member of the transforming acidic coiled coil-containing gene family, TACC3, maps in $4 p 16$, close to translocation breakpoints in multiple myeloma, and is upregulated in various cancer cell lines. Genomics 1999, 58(2):165-70.

44. Ulisse $\mathrm{S}$, et al: Transforming acidic coiled-coil 3 and Aurora-A interact in human thyrocytes and their expression is deregulated in thyroid cancer tissues. Endocr Relat Cancer 2007, 14(3):827-37.

45. Kufer TA, et al: Human TPX2 is required for targeting Aurora-A kinase to the spindle. J Cell Biol 2002, 158(4):617-23.

46. LeRoy PJ, et al: Localization of human TACC3 to mitotic spindles is mediated by phosphorylation on Ser558 by Aurora A: a novel pharmacodynamic method for measuring Aurora A activity. Cancer Res 2007, 67(11):5362-70.

47. Emilsson $V$, et al: Genetics of gene expression and its effect on disease. Nature 2008, 452(7186):423-8
48. Zhang B, Horvath S: A general framework for weighted gene coexpression network analysis. Stat Appl Genet Mol Biol 2005, 4, Article17.

49. Xu X, et al: Quantitative proteomics study of breast cancer cell lines isolated from a single patient: discovery of TIMM17A as a marker for breast cancer. Proteomics 2010, 10(7):1374-90.

50. Li J, et al: Identification of high-quality cancer prognostic markers and metastasis network modules. Nat Commun 2010, 1:34

51. Akavia UD, et al: An integrated approach to uncover drivers of cancer. Cell 2010, 143(6):1005-17.

52. Jornsten $\mathrm{R}$, et al: Network modeling of the transcriptional effects of copy number aberrations in glioblastoma. Mol Syst Biol 2011, 7:486.

53. Cahan $P$, et al: The impact of copy number variation on local gene expression in mouse hematopoietic stem and progenitor cells. Nat Genet 2009, 41(4):430-7.

54. Stranger $\mathrm{BE}$, et al: Relative impact of nucleotide and copy number variation on gene expression phenotypes. Science 2007, 315(5813):848-53.

55. Hastings PJ, et al: Mechanisms of change in gene copy number. Nat Rev Genet 2009, 10(8):551-64.

doi:10.1186/1752-0509-5-121

Cite this article as: Tran et al:: Inferring causal genomic alterations in breast cancer using gene expression data. BMC Systems Biology 2011 5:121.

\section{Submit your next manuscript to BioMed Central and take full advantage of:}

- Convenient online submission

- Thorough peer review

- No space constraints or color figure charges

- Immediate publication on acceptance

- Inclusion in PubMed, CAS, Scopus and Google Scholar

- Research which is freely available for redistribution 